\title{
Landlust und Landfrust
}

\section{Soziale Arbeit in ländlichen Räumen}

„Das Land“ hat in letzter Zeit Konjunktur. Zahlreiche Zeitschriften zeigen in Hochglanz die Vorzüge des Landlebens. Selten fehlen Kaminfeuer, glückliche Kinder malen oder basteln mit ebenso glücklichen und vollkommen ungestressten Eltern in Haus und Garten, die Welt ist heil und offeriert Geborgenheit, Zeit zum Ausruhen und Entspannen. Auch in Romanen ist „das Land“-oder eher „die Provinz" - gegenwärtig wiederholt Gegenstand von Betrachtungen: AutorInnen besuchen ländliche Gegenden und berichten entweder fasziniert oder gelangweilt von ihren Eindrücken und Erlebnissen.

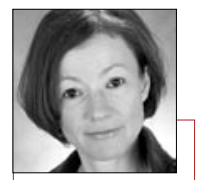

Stefanie Debie *1965

Dr., Professorin für Soziale Arbeit/Kinder, Jugendliche an der HAWK Hildesheim, Holzminden, Göttingen, Fakultät Management, Soziale Arbeit Bauen in Holzminden.

debiel @hawk-hhg.de

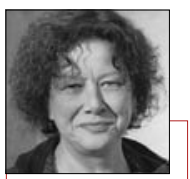

Leonie Wagner *1960

Professorin für Pädagogik und Soziale Arbeit an der HAWKHS Holzminden.

leonie.wagner @hawk-hhg.de
Während die Zeitschriften ungebrochen das Bild der heilsamen Kraft des Landes, den Mythos der Idylle aufgreifen, wird in den literarischen Berichten auch deren Gegenteil Thema: unliebsame Tradition und Enge, verkrustete Strukturen und Aufbegehren Einzelner. Bilder vom Leben auf dem Land waren stets von dieser Ambivalenz geprägt: auf der einen Seite Idylle, auf deren anderen Seite Zurückgebliebenheit, Orte, die man (und vor allem frau) besser schnell verlässt, um die Freuden und Freiheiten der Städte zu genießen - Stadtluft macht frei!

Ländliche Regionen stehen zudem im Zentrum, wenn es um die Problematik von Abwanderung und Überalterung angesichts demographischer Prozesse geht. Dabei muss jedoch zwischen unterschiedlichen Strukturen unterschieden werden. Die „Speckgürtel“ der Großstädte gehören derzeit nicht zu den Bevölkerungsverlierern. Vielmehr sind dies „periphere“ Regionen mit schlechter Verkehrsanbindung und fehlender Wirtschaftsstruktur, aus denen vor allem junge gut ausgebildete Frauen wegziehen. Zurück bleiben ältere und alte Menschen und die Jungen, deren Qualifikationen auf den Arbeitsmärkten der Welt keinen hohen Wert haben. Diese Gruppen haben wiederum unterschiedliche - wenn auch nicht einheitliche - Interessen und Bedürfnisse, die es zu beachten gilt, wenn Soziale Arbeit ihrem Auftrag, Teilhabechancen zu schaffen bzw. zu erhalten und Lebensbedingungen zu verbessern, nachkommen will.

Soziale Arbeit in ländlichen Räumen ist noch nicht in allen Bereichen hinsichtlich ihrer Spezifika und besonderen Herausforderungen untersucht und entwickelt worden. Der Schwerpunkt versammelt deshalb Beiträge, in denen es um diese Besonderheiten geht. Sabine Hering zeigt an Beispielen aus Osteuropa, dass der Blick der Wohlfahrtsgeschichtsschreibung bislang auf die These der Entstehung Sozialer Arbeit in den sich entwickelnden Industrieregionen und den entsprechenden Problemfeststellungen verengt ist. Daran knüpft die Frage an, ob der „welfare mix“ nicht neben politisch-ideologischen und zivilgesellschaftlichen auch maßgeblich auf infrastrukturelle Rahmenbedingungen zurückgeht.
Leonie Wagner stellt in ihrem Beitrag relevante Spannungsfelder vor, die die Ausgestaltung Sozialer Arbeit in ländlichen Räumen beeinflussen (sollten). Diese Spannungsfelder werden in den folgenden Beiträgen auf der Grundlage der Betrachtung spezifischer Lebenslagen und damit verbundener Bedürfnisse und infrastruktureller Probleme weiter differenziert. Anke Strube zeigt die Herausforderungen für soziale Altenarbeit und plädiert für an ländliche Räume angepasste quartiersbezogene Ansätze, die die Interessen und Bedürfnisse der dort lebenden älteren Menschen aufnehmen und deren Teilhabe erhalten und verbessern.

Björn Redmann kritisiert den Abbau der Ressourcen für Jugend(sozial)arbeit in ländlichen Räumen, die eine fachlich und rechtlich angemessene Angebotsstruktur zunehmend verunmöglichen - insbesondere in ostdeutschen ländlichen Regionen. Er spricht sich für eine parteiliche und streitbare Jugend-(sozial-)arbeit aus, der es nicht nur um Anpassung und Reparaturleistungen geht. Stefanie Debiel stellt am Beispiel von Mehrgenerationenhäusern und weniger institutionalisierten Projekten intergenerationelle Angebote vor, die die Chance bieten, vorhandene Ressourcen zu vernetzen und zu bündeln bzw. gerade durch Vernetzung zwischen den Generationen neue Potenziale zu schaffen.

Die AutorInnen kommen zu dem Schluss, dass die Anforderungen an Soziale Arbeit in ländlichen Räumen sich zwar nicht grundlegend und prinzipiell von denen in Städten unterscheiden, sie heben aber hervor, dass es darum gehen muss, sozialräumlich zu arbeiten und dabei vor allem die Interessen und Bedürfnisse der auf dem Land lebenden Menschen genau zu untersuchen, vorhandene Rahmenbedingungen zu erhalten bzw. zu verändern oder zurückzuerobern oder gar neue zu schaffen. 